\title{
Bioanalysis
}

\section{WRIB Poster awards winners 2020}

\author{
Zifeng Mai*,1, David Escobar**,2, Shuo Wang***,2 \& Guangnong Sunny Zhang****,3 \\ ${ }^{1}$ Frontage Laboratories, 700 Pennsylvania Drive, Exton, PA 19341, USA \\ ${ }^{2}$ Navigate BioPharma Services, 1890 Rutherford Road, Carlsbad, CA 92008, USA \\ ${ }^{3}$ AstraZeneca, 35 Gatehouse Drive, Waltham, MA 02451, USA \\ *Author for correspondence: zmai@frontagelab.com \\ **Author for correspondence: david.escobar@navigatebp.com \\ ***Author for correspondence: shuo.wang@navigatebp.com \\ $* * * *$ Author for correspondence: guangnong.zhang@astrazeneca.com
}

First draft submitted: TBC; Accepted for publication: 21 October 2020; Published online: 13 November 2020

Keywords: WRIB $\bullet$ bioanalysis $\bullet$ biomarkers $\bullet$ immunogenicity $\bullet$ gene therapy $\bullet$ cell therapy $\bullet$ vaccine

The 14th edition of the workshop on recent issues in bioanalysis (14th WRIB) was held virtually between 15-29 June 2020 with an attendance of over 1000 professionals from pharmaceutical/biopharmaceutical companies, biotechnology companies, contract research organizations and regulatory agencies worldwide. The 14th WRIB included three main workshops, seven specialized workshops that together spanned 11 days to allow exhaustive and thorough coverage of all major issues in bioanalysis, biomarkers, immunogenicity and gene and cell therapy. High quality, better compliance to regulations and scientific excellence are always the foundations of this workshop. Moreover, a comprehensive vaccine assays track; an enhanced cytometry track and updated industry/regulators' consensus on bioanalytical method validation (BMV) of biotherapeutics by mass spectrometry (hybrid assays, LCMS/MS and HRMS) were special features in 2020. As in previous years, this year's WRIB continued to gather a wide diversity of international industry opinion leaders and regulatory authority experts working on both small and large molecules to facilitate sharing, reviewing, discussing and agreeing upon best approaches aimed to achieve scientific excellence and increase regulatory compliance on bioanalytical issues.

Bioanalysis and Bioanalysis Zone are very proud to continually supporting the WRIB Poster awards, and we feature the profiles of the authors of the winning posters. Visit www.bioanalysis-zone.com to see the winning posters in full.

\section{Introduction}

The 14th edition of the workshop on recent issues in bioanalysis (14th WRIB) was held virtually between 15-29 June 2020 with an attendance of over 1000 representatives from pharmaceutical/biopharmaceutical companies, biotechnology companies, contract research organizations and regulatory agencies worldwide. The 14th WRIB included three main workshops, seven specialized workshops that together spanned 11 days to allow exhaustive and thorough coverage of all major issues in bioanalysis, biomarkers, immunogenicity, gene therapy, cell therapy and vaccine. Moreover, a comprehensive vaccine assays track; an enhanced cytometry track and updated industry/regulators' consensus on bioanalytical method validation (BMV) of biotherapeutics by mass spectrometry (hybrid assays, LC-MS/MS and HRMS) were special features in 2020. As in previous years, this year's WRIB continued to gather a wide diversity of international industry opinion leaders and regulatory authority experts working on both small and large molecules to facilitate sharing, reviewing, discussing and agreeing upon best approaches aimed to achieve scientific excellence and increase regulatory compliance on bioanalytical issues. Bioanalysis and Bioanalysis Zone are very proud to continually supporting the WRIB Poster awards, and we feature the profiles of the authors of the winning posters. Visit www.bioanalysis-zone.com to see the winning posters in full. 


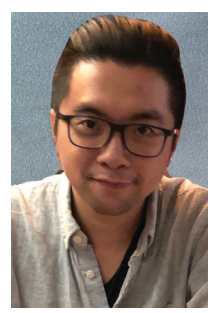

\section{Bioanalytical drug tolerance limit prediction \& optimization model developed through machine learning with TensorFlow \\ Zifeng Mai (Frontage Laboratories, USA)}

zmai@frontagelab.com

I am the Associate Director of Biologics Services at Frontage Laboratories. Our work covers immunogenicity, PK and biomarker and specializes in anti-drug-antibody (ADA) and Nab assays. Prior to joining Frontage, I worked at the Yale School of Medicine, responsible for multiple myeloma relapse mechanism and melanoma cell profiling research. After Yale, I worked at Bristol-Myers Squibb and participated in assay development in in vitro and ex vivo studies using primary cells and tumor tissues. Since joining Frontage, I started as a scientist, and worked my way up to leading a team of eight senior and junior scientists.

\section{Please can you explain about your role at Frontage?}

I am the Associate Director of Biologics Services at Frontage Laboratories. Frontage is a CRO company providing integrated, science-driven, product development services throughout the drug discovery and development process. Its headquarter is in Exton, Pennsylvania, with other sites in the USA and China. Our group works on immunogenicity, $\mathrm{PD}$ and biomarker and specializes in $\mathrm{ADA}$ and $\mathrm{Nab}$ assays. My responsibilities include leading the team to deliver assay development, validation and sample analysis and managing the overall planning, execution and quality of assigned MD/MV projects. Troubleshooting is also a major part of my job. I am a passionate scientist and leader who is always craving for opportunities to combine bodies of knowledge across disciplines, troubleshoot and make them useful to me.

\section{How does it feel to win the 14th WRIB Poster award?}

It was a great honor and a privilege to be considered for this Poster award among the many excellent ideas and works. To be honest I did not expect this since it was only a rough idea at the beginning, which I was not sure if it would work. The good results gave me confidence, and this award further urges me to dedicate more time on this project and make my contribution to the field of ADA assay development.

\section{Describe your experience of virtually presenting your work?}

It was quite different, but fun. Instead of seeing and actually talking with professionals in a physically gathered location, virtually presenting adds an extra layer and requires me to think about how to engage my audience without the in-person interactions. I hope it was fun and inspiring to watch my presentation!

Tell us a little about the work on which the Poster was based?

This project was based on two distinct areas - one is the ADA assay development, the other is machine learning. The data used in this project came from our daily assay development.

What were the key conclusions from your research?

First and foremost, this project proved that machine learning can be, and should be considered for ADA drug tolerance limit prediction. Though machine learning has been applied in many subfields in the pharmaceutical world, it was understudied in this particular area. Second, the results demonstrated that machine learning can maximize the assay drug tolerance limit in a faster and easier way than what we are doing. In short, this project starts the conversation of a new perspective and approach. I would say one major takeaway would be that never limit ourselves to what we are used to doing, and always think about how we can do things differently and more efficiently.

\section{What are you looking forward to working on over the next year?}

I will continue to explore the possibility of machine learning applications in the bioanalytical field. And of course, I will continue to work on this project by adding more data into the model and finetune the machine learning model in predicting drug tolerance limit. 


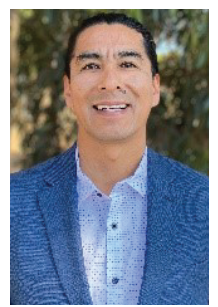

\section{Pharmacodyanamic analysis of crizanlizumab by surface plasmon resonance: a case for implementing unique analytical platforms in clinical trial} David Escobar (Navigate BioPharma Services, USA) david.escobar@navigatebp.com

Originally from La Paz, Bolivia emigrated to the US 32 years ago and I received my BS in molecular biology from San Diego State University in 1998. I have had the fortune to have interacted and learned from talented scientists with huge hearts. I like to take this opportunity to thank you all for your teachings. I incurred into the bioanalytical world in 2006 working as an analyst in Joe Smolec's lab testing samples from PK and immunogenicity studies. Eleven years and countless of validations later, in 2017, I joined Navigate BioPharma Services, where I am currently the manager for the ligand binding assay technology (LBAT) group. The journey continues looking forward to new challenges in unprecedented times.

\section{Shuo Wang (Navigate BioPharma Services, USA)} shuo.wang@navigatebp.com

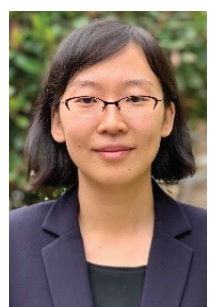

I am a motivated and goal-driven scientist with deep expertise in biomolecular interaction characterization using biophysical and biochemical approaches. I obtained my $\mathrm{PhD}$ degree in biophysical chemistry studying the energetic and molecular basis of regulating transcription factors by DNA targeting small molecules. During my time working in industry, I have worked on projects in different stages of the drug discovery pipeline from the initial library screening, lead generation and optimization, to clinical trial testing in different phases. What interests me the most is the mechanism of action of drug candidates, and how we can design biomolecular assays to provide physiological relevant answers. I am also a big believer of teamwork because teamwork makes the dream work.

\section{Please can you explain about your role at Navigate BioPharma?}

We work in the LBAT group at Navigate BioPharma. David is the group lead and Shuo is a senior scientist. Our group has extensive experience in developing and validating immunoassays (e.g., ELISA, SIMOA, surface plasmon resonance $[\mathrm{SPR}]$ etc.) and also conducting testing for clinical trials in a regulated environment.

\section{How does it feel to win the 14th WRIB Poster award?}

Surreal is the first adjective that came to our mind. It is definitely an honor to win the Poster award in such a prestigious conference/workshop. We see this award as the positive impact that our work is making on the LBA community and on patients' lives. Our goal is the same as our company Navigate BioPharma's motto, which is to be the pioneers, partners and pathfinders.

\section{Describe your experience of virtually presenting your work?}

David: Tedious, strange and at the same time almost normal. As most scientists are accustomed to present in front of people, producing a video, I feel, should have been fairly straight forward. It was the opposite. It took several tries to get the words and tone 'right' for the video to flow. This experience has given me a different sense of appreciation for the work actors or movie producers do.

\section{Tell us a little about the work on which the Poster was based?}

The assay itself saw its birth in a laboratory in Oklahoma. Our task, once the assay was transferred to Navigate, was to further optimize it and, of course, validate it. Shuo being the SPR subject matter expert and myself having many years under my belt validating immunoassays, were recruited and brought together to onboard this assay at Navigate BioPharma for clinical testing use.

Crizanlizumab is the first and only US FDA approved monoclonal antibody (marketed as Adakveo ${ }^{\circledR}$ ) that reduces pain crises in adults and pediatric patients ( $\geq 16$ years) with sickle cell disease. Our SPR based PD assay mimics the in vivo biological interactions of crizanlizumab with P-selectin and its glycoprotein ligand-1 on red blood cells. We presented in the poster the assay optimization and systematic validation in order to share our experience using SPR in a LBA clinical trial setting. 
What were the key conclusions from your research?

We were able to validate an assay using SPR technology, which is lesser known in the world of LBA clinical trial testing. The assay itself generated data for a coprimary end point of a clinical trial that supported the registration of crizanlizumab.

\section{What are you looking forward to working on over the next year?}

The LBAT group is and will be supporting trial testing for ongoing crizanlizumab studies. We have also recently started to work on developing COVID-19 serology assays. Our total antibody assay should be available by midsummer 2020.

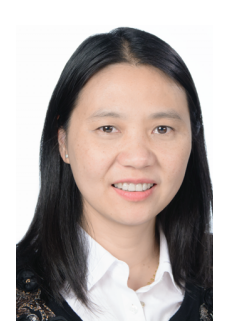

\section{Quantitation of released \& total AZD4320 from nanomedicine dendrimer by LC-MS/MS}

Guangnong (Sunny) Zhang (AstraZeneca, USA)

guangnong.zhang@astrazeneca.com

Guangnong (Sunny) Zhang is a senior scientist in early oncology R\&D DMPK at AstraZeneca in Boston, Massachusetts. Sunny obtained her PhD degree in pharmacognosy from China Pharmaceutical University (Nanjing, China). Prior to joining AstraZeneca in 2018, Sunny spent 11 years as a principal scientist/study director at several CRO's including Olon Biosciences, Medpace, Inc. and Charles River Laboratories. Her responsibilities in those roles were to conduct and oversee regulated bioanalysis for preclinical and clinical studies. Sunny's expertise lies in high-throughput discovery bioanalysis, regulated preclinical and clinical bioanalysis of small molecule drugs, peptides and biomarkers in complex biological matrices. Her experience also covers CRO outsourcing and study management.

\section{Please can you explain about your role at AstraZeneca?}

I am a senior scientist working in the Early Oncology R\&D DMPK department at AstraZeneca. In my role, I provide bioanalytical method development and analysis support for in vivo $\mathrm{PK}$ and $\mathrm{PD}$ samples across the global oncology portfolio. I develop high throughput bioanalytical methods, conduct quantitative LC-MS/MS assays, and perform noncompartmental PK analysis. As part of the oncology DMPK group, I interact with global and local DMPK members, project teams, as well as collaborating with vendors. All for the purposes of executing DMPK strategy to discover lifesaving cancer medications.

\section{How does it feel to win the 14th WRIB Poster award?}

It was a great honor and a privilege to receive this award in recognition of our nanomedicine bioanalysis work. We are proud that the methods we have developed were received positively by the bioanalytical community. Additionally, we are proud that this recognition gathers credibility for AstraZeneca as an innovator in the field of nanomedicines and their bioanalysis.

I would like to thank all the great scientists from the BCL-2/Bcl-xL project and advanced drug delivery teams at AstraZeneca. And also a special thanks to our external partner Starpharma for their contributions toward this work. WRIB is a highly respected conference and being recognized among so many excellent posters presented is a career highlight for me.

\section{Describe your experience of virtually presenting your work?}

I very much enjoyed my virtual experience at WRIB. Unlike in-person presentations, I feel the audience had a greater opportunity to experience a personalized presentation for as many posters as they wished. Additionally, this format seems to offer a more holistic visual-audio experience. Many thanks to the organizers of the WRIB conference for providing such a quality platform.

Although we are disappointed to not meet colleagues in-person, we were able to listen to all of the great talks from the comfort and safety of our homes. Having the ability to listen to interesting talks a second or third time was also quite valuable. This virtual format also enabled more effective and transparent engagement with colleagues sharing interest in our work. This is quite remarkable given that it occurred during a global pandemic. The 14th WRIB conference was truly a success that overcame the barriers imposed by COVID-19. 
Tell us a little about the work on which the Poster was based?

Novel drug delivery systems are an innovative approach to improve the pharmaceutical properties of a candidate drug. But reliable measurement of the released and 'total' (released + nanomaterial-associated) drug concentration is critical to understand the drug exposure and $\mathrm{PK} / \mathrm{PD} /$ efficacy relationships. The poster gives an overview of the key challenges and approaches for dendrimer based nanomedicine bioanalysis.

More specifically, we offered bioanalytical methods to quantitate AZD4320, a potent dual Bcl-2/Bcl-xL inhibitor developed for the treatment of hematologic and solid tumors. This innovative drug delivery platform composed of AZD4320 and a PEGylated dendrimer, was developed to improve solubility and therapeutic margin. We identified key challenges and established general guidance to selectively and accurately quantitate 'released' and 'total' drug concentrations in biological matrices.

\section{What were the key conclusions from your research?}

Nanomedicines have unique bioanalytical challenges that require innovative solutions. A general 'NanoSToRe' (nanoparticle strategy using 'totals' and 'released' assay) was created in this poster. The overall concepts may serve as a paradigm for future nanomedicine bioanalysis. Where the key principles presented were to stabilize and subsequently physically separate nanomedicine material from plasma. Here, robust methods were developed by leveraging temperature, acidity and liquid-liquid extraction approaches. The presented method(s) demonstrated high selectivity, sensitivity, linearity, accuracy and precision.

\section{What are you looking forward to working on over the next year?}

Innovation in the oncology therapy field is both diverse and rapidly evolving. I look forward to the unique bioanalytical challenges that they will present. LC-MS/MS method development for challenging chemical space like - $\log \mathrm{D}$ molecules, new modalities, advanced drug-delivery systems and PROTAC-based therapies are all on the horizon and will offer numerous welcomed opportunities.

\section{Disclaimer}

The opinions expressed in this interview are those of the interviewee and do not necessarily reflect the views of Newlands Press Ltd.

\section{Financial \& competing interests disclosure}

The interviewees have no relevant affiliations or financial involvement with any organization or entity with a financial interest in or financial conflict with the subject matter or materials discussed in the manuscript. This includes employment, consultancies, honoraria, stock ownership or options, expert testimony, grants or patents received or pending, or royalties.

No writing assistance was utilized in the production of this manuscript. 
